\title{
The minimization of ac phase noise in interferometric systems
}

\author{
Filinski, Ignacy; Gordon, R A
}

Published in:

Review of Scientific Instruments

Link to article, DOI:

$10.1063 / 1.1145120$

Publication date:

1994

Document Version

Publisher's PDF, also known as Version of record

Link back to DTU Orbit

Citation (APA):

Filinski, I., \& Gordon, R. A. (1994). The minimization of ac phase noise in interferometric systems. Review of Scientific Instruments, 65(3), 575-586. https://doi.org/10.1063/1.1145120

\section{General rights}

Copyright and moral rights for the publications made accessible in the public portal are retained by the authors and/or other copyright owners and it is a condition of accessing publications that users recognise and abide by the legal requirements associated with these rights.

- Users may download and print one copy of any publication from the public portal for the purpose of private study or research.

- You may not further distribute the material or use it for any profit-making activity or commercial gain

- You may freely distribute the URL identifying the publication in the public portal

If you believe that this document breaches copyright please contact us providing details, and we will remove access to the work immediately and investigate your claim. 


\title{
The minimization of ac phase noise in interferometric systems
}

\author{
I. Filinski and R. A. Gordon \\ Physics Department, The Technical University of Denmark, B. 309, DK-2800 Lyngby, Denmark
}

(Received 27 September 1993; accepted for publication 9 December 1993)

A simple step-by-step procedure, including several novel techniques discussed in the Appendices, is given for minimizing ac phase noise in typical interferometric systems such as two-beam interferometers, holographic setups, four-wave mixers, etc. Special attention is given to index of refraction fluctuations, direct mechanical coupling, and acoustic coupling, whose importance in determining ac phase noise in interferometric systems has not been adequately treated. The minimization procedure must be carried out while continuously monitoring the phase noise which can be done very simply by using a photodiode measurement of the interferometer output. Supplementary measurements using a microphone and accelerometer will also be helpful in identifying the sources of phase noise. Emphasis is placed on new techniques or new modifications of older techniques which will not usually be familiar to most workers in optics. Thus, the necessity of eliminating the effects of index of refraction fluctuations which degrade the performance of all interferometers is pointed out as the first priority. A substantial decrease of the effects of all vibrating, rotating, or flowing masses (e.g., cooling lines) in direct contact with the optical table will also have to be carefully carried out regardless of the type of interferometric system employed. It is recommended that this be followed by a simple, inexpensive change to a novel type of interferometer discussed in Appendix A which is inherently less sensitive to mechanical vibration. Such a change will lead to a reduction of both low-frequency and high-frequency ac phase noise by more than an order of magnitude and can be carried out for all interferometers with the exception of multiple pass optical systems and high-resolution FFT spectrometers. It is pointed out that most homemade air bladder vibration isolators are used incorrectly and do not provide sufficient reduction in the contribution of floor vibrations to phase noise. Several simple trampoline-type air bladder vibration isolator systems are described which are comparable in performance to commercial systems. With the exception of very nonrigid or undamped optical tables, the dominant source of ac phase noise at this point will usually be due to acoustic coupling to the optical components and mounts themselves. This means not only that the optical components and mounts must be rigid but that the mechanical coupling between the table and the mounts, as well as the coupling between the mounts and components themselves, be as rigid as possible. An additional damping of optical mounts beyond that generally found in commercial mountings will also have to be carried out to obtain a further reduction of phase noise. A simple damping technique employing an additional mass and an intermediate damping layer is described which will significantly improve the performance of both homemade and commercial optical mounts. Similar damping techniques which are especially suitable for homemade optical tables and breadboards are also considered.

\section{INTRODUCTION}

Interferometric techniques are at the heart of many of the most sensitive measurements in Science and Technology. Unfortunately, a more widespread application of such techniques is often limited by the common false impression that interferometric techniques are difficult or expensive. Such false impressions are all too often engendered by typical first encounters with notoriously vibration-sensitive interferometric systems, such as the Michelson interferometer commonly found in student laboratories. In reality, interferometric techniques are frequently the essence of simplicity as can be strikingly demonstrated by the almost trivial production of extremely stable white light fringes using an ordinary incandescent light bulb together with two mirrors and a plane-parallel glass plate mounted on a desk table top, as discussed in this article. ${ }^{1,2}$

With this point in mind, we describe here a carefully tested set of simple, inexpensive procedures based on new techniques and modifications of older techniques, which can be readily applied to build reliable, sensitive interferometric systems. ${ }^{3}$ In order to give a uniform presentation, all of the procedures recommended here have been personally checked by the authors over a wide range of laboratory conditions. The primary purpose is to enable the reader to carry out a reliable effective minimization of ac phase noise as quickly, simply, and inexpensively as possible.

\section{GENERAL REMARKS}

Interferometer output instabilities are due to fluctuations in the phase difference between interfering beams. In this paper very slow ac variations of the phase, corresponding to frequencies of a fraction of a $\mathrm{Hz}$, will not be considered. It is also assumed that the performance of the interferometer system is limited only by ac phase noise and not 


\begin{tabular}{|c|c|c|c|}
\hline $\begin{array}{l}\text { NOISE } \\
\text { SOURCES }\end{array}$ & $\begin{array}{l}\text { NOISE } \\
\text { TYPES }\end{array}$ & $\begin{array}{l}\text { IRANSMISSION } \\
\text { (source damping, } \\
\text { path attenuation) }\end{array}$ & $\begin{array}{l}\text { MECHANICAI. } \\
\text { RESPONSE }\end{array}$ \\
\hline
\end{tabular}

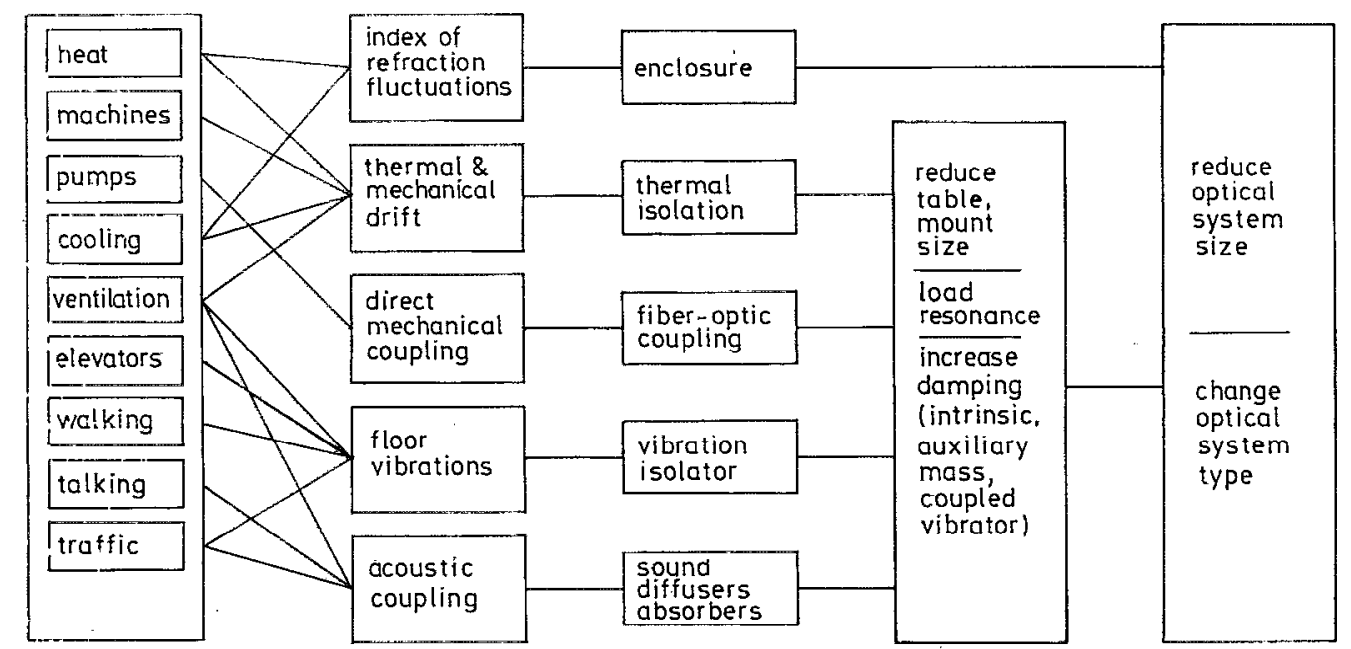

FIG. 1. A graphic depiction of the different links in the source-to-signal chain and the various types of ac phase noise for common sources of ac phase noise as discussed in the text: As can be seen, the noise minimization procedure should proceed from right to left in the figure as discussed in Secs. $V$ and VI.

by any imperfections in the optical system such as nonplanarity or roughness of optical surfaces, finite curvature of the interfering beams, different amplitudes of the interfering beams, etc.

\section{TYPES OF ac PHASE NOISE}

As can be seen from Fig. 1, the sources responsible for ac phase noise are coupled to the optical response in an extremely large number of different ways which can be conveniently grouped into two different general classes depending on whether they are induced by a change in the geometrical path, or are due to fluctuations in the refractive index.

\section{A. Index of refraction fluctuations}

The main sources of index of refraction fluctuations appear as thermally induced variations in the optical density of the air or the fluids in the optical path leading to both local fluctuations and flows, e.g., convection currents. This is generally the dominant source of ac phase noise up to $10-20 \mathrm{~Hz}$ with an amplitude on the order of one interference fringe. ${ }^{4-7}$

\section{B. Direct mechanical coupling}

This type of ac noise appears as vibrations induced in the optical system by vibrating masses in direct mechanical contact with the optical table and mounts, such as transformers, motors, air or water cooling systems commonly employed with lasers, pulsed lasers, etc. This is frequently the largest unsuspected source of mechanically induced phase noise over the entire range of frequencies of mechanical motion even up to several $\mathrm{kHz}^{8,9}$

\section{Floor vibrations}

Such vibrations are transmitted to the optical system through optical table supports leading to an ac variation in the geometric dimensions of the optical system. ${ }^{10-14}$ The spectrum of such noise will frequently contain sizeable sinusoidal resonance components up to several hundred $\mathrm{Hz}$ in addition to that characteristic of ground (seismic) vibrations which are usually appreciable only below $40 \mathrm{~Hz}$. Thus, floor vibrations will generally consist of a broad band of spectral components between $1 \mathrm{~Hz}$ and $1 \mathrm{kHz}$ originating from ventilation systems, air conditioning, vacuum pumps, motors, structural vibrations, etc., plus a random component due to passing cars, elevator motors, workshop machines, etc.

\section{Acoustic coupling}

Acoustic coupling appears in the form of vibrations of the optical system induced by the acoustic noise generated by ventilators, air conditioning, vacuum pumps, structural vibrations (e.g., walls, floors, partitions), music, speech, street noise, etc. over a frequency range from $\sim 10$ to $\sim 1000 \mathrm{~Hz}$ in the form of sharp coherent resonances (of very large number) as well as transient excitations. ${ }^{8,9,15,16}$. The ac phase noise due to acoustic coupling will usually be a sizeable fraction of an interference fringe and can be large at lower frequencies where the acoustic flux, although inaudible to the human ear, might still introduce large amplitude vibrations. The importance of acoustic coupling as the dominant residual source of phase noise in most interferometric systems is not commonly appreciated. 


\section{E. Thermal and mechanical drift}

Such drift appears as slow (minutes to hours per interference fringe) generally monotonic changes in the optical phase with a large total drift amplitude on the order of several fringes. Such ac phase noise results from changes in the temperature and the mechanical tension over the optical system and results from mechanical backlash, local heating, adjustments of optical components, film mounting, etc. The effects of such sources on ac phase noise can be minimized by the choice of high-quality optical tables, mounts and components and/or by careful attention to the thermal and mechanical environment. In many cases, it may be easiest to change to a double-beam circular interferometer (DBCI) optical system (see Appendix A) or to use active stabilization of the optical path of the interferometer-rather than minimizing thermal and mechanical drift directly. ${ }^{17-19}$

\section{SOURCE-TO-SIGNAL CHAIN}

Fluctuations, $\delta n$, in the index of refraction, $n$, of the gas within the optical path can be written very simply as

$$
\delta n \approx(1-n) \frac{\delta T}{T}=-\left(79 \times 10^{-6}\right) \frac{\delta T}{T^{2}} P_{\text {air }}(\text { mbar }),
$$

where $\delta T$ represents the fluctuations in the temperature, $T$ in degrees $K$, and where the expression on the extreme right side of Eq. (1) applies to air at the pressure $P_{\text {air }}{ }^{7,20}$ If liquids are present in the optical path, the expression for $\delta n$ will be more complicated. ${ }^{21}$

The four remaining types of ac phase noise are due to vibrations of the optical system. The total ac phase noise $(\Delta \phi)_{\text {rms,mech }}$ for such mechanically induced noise sources can be written as the sum of the product of four factors which are different for each source of ac phase noise

$$
(\Delta \phi)_{\mathrm{rms}, \mathrm{mech}}=R_{\mathrm{opt}} \sum A_{i} T_{i} R_{\mathrm{mech}, i},
$$

where the summation is taken over all mechanically induced noise sources. Here $A_{i}$ is the amplitude of each noise source in appropriate units, $T_{i}$ is the fraction $\left(0<T_{i}<1\right)$ of the amplitude of each source which is transmitted to the interferometric system after source damping and path attention, $R_{\text {mech }, i}$ is the mechanical response giving the change in the geometrical path difference between optical components caused by vibrations of the interferometric system, and lastly $R_{\text {opt }}$ is the optical response giving the change in the optical path difference due to the vibrationally induced change in the geometrical path difference. ${ }^{22}$ It should be emphasized that $R_{\text {mech, } i}$ will be dominated by resonances of the mechanical system shown in Fig. 2 with only relatively small contributions from nonresonant vibrations or transient vibrational excitations under normal experimental conditions. In practice, all of the factors in Eqs. (1) and (2) can vary significantly with especially large variations up to one or two orders of magnitude in the factors $R_{\text {mech }, l}$ and $R_{\text {opt }}$ commonly attainable. Thus, a reduction in the optical response should be implemented as early as possible followed by a reduction in the mechanical

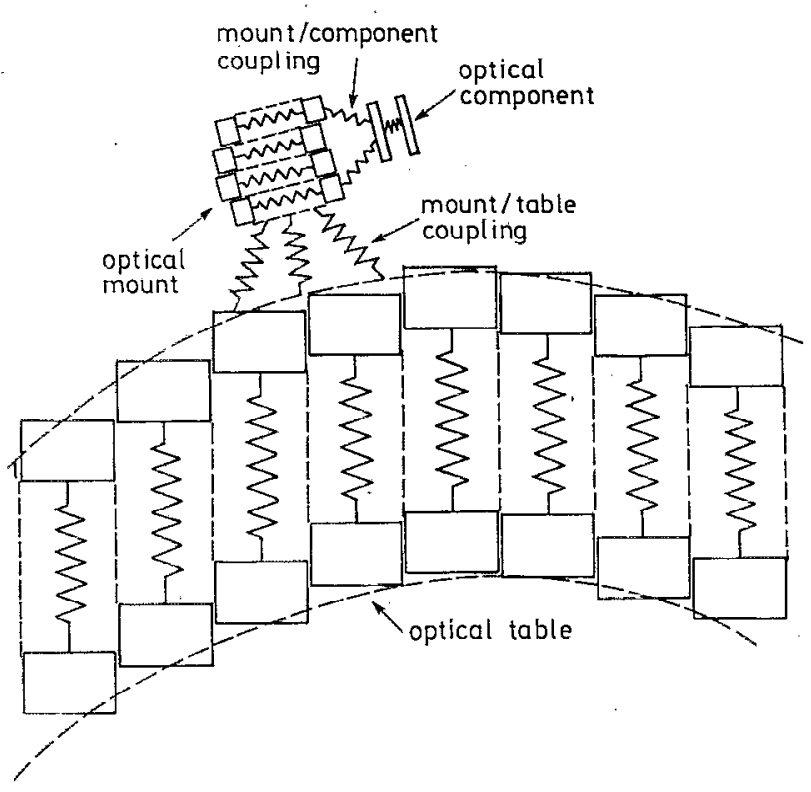

FIG. 2. Schematic representation of the different types of mechanical vibrations which can contribute to ac phase noise. The phase noise due to such vibrations will be dominated by the resonant response(s) of each of the vibrational motions depicted in the figure which should be minimized by increasing the stiffness and damping of all of the springs as much as possible. It is important to note that with the exception of very nonrigid or lightly damped optical tables the ac phase noise will be dominated by the vibrations of optical mounts under normal experimental conditions.

response, and lastly by a reduction in the factors $T_{i}$ and $A_{i}$. A modest straightforward reduction of several different factors (especially $R_{\text {opt }}$ and $R_{\text {mech }, i}$ ) in the source-to-signal chain will almost always be more effective than a timeconsuming or expensive state-of-the-art decrease in only one of the factors appearing in Eq. (2).

\section{INSTRUMENTATION}

We recommend the following instrumentation to identify and measure the sources of ac phase noise. If it is not possible to modify the actual interferometric system of interest to give an output which is simply related to the ac phase noise, a simple Michelson interferometer should be built covering a large portion of the optical table using the same optical components as those to be employed in the final interferometric system. ${ }^{20,23,24}$ The difference in the length of the two arms of the interferometer should be kept small (preferably no more than $1-2 \mathrm{~cm}$ ) in order not to reduce fringe contrast. A $\mathrm{He}-\mathrm{Ne}$ laser (preferably polarized) should be used as the light source. ${ }^{25}$ The interferometer output can be projected onto a screen for qualitative measurements or onto a silicon photodiode for more quantitative measurements, preferably such that the entire photodiode detector area is covered by a single interference fringe. A signal proportional to the short-circuit current of the photodiode should be connected to one of the channels of a two-channel $\mathrm{dc}$ coupled oscilloscope. A digital oscilloscope is especially convenient allowing both rapid and slow changes in the optical phase difference to be measured. The interferometer should be adjusted such that the 
photodiode output lies halfway between the maximum and minimum photodiode outputs, $I_{\max }$ and $I_{\min }$, respectively, corresponding to maxima and minima of the interference pattern. (If this is not possible because of very large ac phase noise, then one or more steps of the standard procedure will have to be carried out before any measurements of the size of the ac phase noise can be made.) It is possible to estimate the change in the optical phase difference, $\phi(t)$, relative to the value of the phase at the halfway intensity position, from the difference in the photodiode outputs, $I\left(t_{1}\right)$ and $I\left(t_{2}\right)$, at two different times, $t_{1}$ and $t_{2}$, respectively, since

$$
\begin{aligned}
\frac{I\left(t_{1}\right)-I\left(t_{2}\right)}{\left(I_{\max }-I_{\min }\right) / 2} & =\sin \phi\left(t_{1}\right)-\sin \phi\left(t_{2}\right) \\
& \cong \phi\left(t_{1}\right)-\phi\left(t_{2}\right)=\Delta \phi .
\end{aligned}
$$

The expression on the extreme right side of Eq. (3) is valid for small changes in the phase and can be conveniently measured from the peak-to-peak amplitude of $I\left(t_{1}\right)-I\left(t_{2}\right)$ observed on the oscilloscope.

An alternative method of estimating small changes in the phase, which is also applicable if an oscilloscope is not available, is to measure the fringe contrast ratio $C$, which is simply related to the rms change in the phase $(\Delta \phi)_{\mathrm{rms}}$,

$$
\begin{aligned}
& C=\frac{I_{\text {max }, \text { rms }}}{I_{\text {min,rms }}}, \\
& (\Delta \phi)_{\mathrm{rms}} \cong \sqrt{4 / C}
\end{aligned}
$$

assuming the light intensity is approximately equal in both arms of the interferometer. Measurements of the amplitude and frequency spectrum of floor and optical table vibrations (both vertical and horizontal) can be carried out using any convenient vibration transducer (accelerometer, velocimeter, gramophone pickup, etc.) capable of detecting accelerations on the order of $1 \mu \mathrm{g}$ from $\sim 5 \mathrm{~Hz}$ to $1 \mathrm{kHz}$, where $g$ is the acceleration of gravity. ${ }^{26,27}$ Vibrations of the optical table and optical components can be excited using a sinusoidal generator (or, even better, a sweep-frequency generator) and HiFi loudspeaker placed close to the optical table or by using a small HiFi earphone with a good low-frequency response fixed directly to the optical table or mounts. ${ }^{28}$ The acoustic flux can be measured by a HiFi microphone with a good low-frequency sensitivity. A spectrum analyzer, when available, can also be recommended in order to simplify measurements of the frequency response of the optical and mechanical response of the optical system. It is worth emphasizing the importance of the photodiode output as direct measure of the phase noise due to all changes in the optical path difference. The accelerometer and microphone are extremely useful in identifying the contributions of the various vibrational noise sources to the total phase noise due to eigenmode vibrations of the optical system as discussed in Sec. VII.

\section{STANDARD PROCEDURE}

The experience of the authors has shown that most requirements for ac phase noise reduction can be met by the standard procedure described below. The standard procedure will also be a necessary prerequisite for workers desiring a further reduction of ac phase noise with the help of the supplementary procedure described in the next section.

It will be helpful for workers who have a choice, to select a relatively low-noise location preferably in the cellar of a laboratory building which is far away from elevators, central heating units, workshops, pumping stations, ventilator ducts, noise water pumps, air conditioning, etc. Laboratory buildings away from highways, railroads, auditoriums, power stations, or other sources of acoustic and vibrational noise are also to be preferred whenever possible.

In order to carry out the standard procedure it will be necessary to measure the ac phase noise directly since it will otherwise be almost impossible to monitor the effect of the various steps in the standard procedure, or to know when the ac phase noise, $(\Delta \phi)$, has been reduced to an acceptable level. This should be done, whenever possible, by measuring the phase noise in the actual optical system of interest. For most interferometric systems this can be easily done using a simple photodiode output. This can, for example, be done for holographic systems by rigidly gluing a small plane mirror to a standard holographic object and using a beam splitter to obtain two nearly parallel interfering beams. For optical systems which can not be readily modified to give a phase difference, e.g., laser cavities, it will be necessary to build a simple Michelson interferometer as noted in the previous section. In this case, measurements of the ac phase noise in the Michelson interferometer can be used to provide an estimate of the improvement in the stability of the original interferometric system.

We now present a list of simple steps in approximately increasing order of difficulty all of which will significantly reduce ac phase noise and all of which should be carried out in a straightforward step-by-step cookbook manner.

(a) Remove all heat (cold) sources or objects differing by even a few degrees from room temperature from locations within or close to the optical cavity of the interferometer. ${ }^{29}$

Unsuspected temperature gradients will frequently be generated in temperature-stabilized intracavity FabryPerot etalons, temperature-tuned frequency doubling crystals, cryostats and ovens in the optical system, etc. In such cases, the index of refraction fluctuations can be reduced by keeping the temperature of all parts of the laser or interferometer cavity and the external optical system as close to. room temperature as possible. Another solution will be to thermally isolate the heated (or cooled) object as much as possible from the rest of the optical system (e.g., with the help of a double-window system) and/or to use helium gas within the optical system leading to smaller fluctuations in the refractive index, viz. Eq. (1). In similar fashion, the use of a cold finger to cool samples and other parts of the optical system instead of immersing them in cryogenic liquids will eliminate index of refraction fluctuations due to bubble cavitation. ${ }^{30,31}$ If immersion in cryogenic liquids cannot be avoided, then special attention will 
have to be given to the coupling of vibrations from mechanical pumps used to lower the temperature or reduce bubble cavitation.

(b) Build an enclosure around the interferometer cavity with a minimum number of openings each of which should be as small as possible without restricting the ability to couple the light beam into or out of the interferometer. ${ }^{32}$

The enclosure need not be airtight and can be constructed very simply and quickly using a tent-like construction built from styrofoam, cardboard, plastic, transparent plastic foil, etc.

(c) All acoustic or mechanical sources of sound and vibration which are in direct physical contact with the optical table should be removed or turned off. Such sources include cooling fans, water pumps, vacuum pumps, water pipes, etc. as discussed in Sec. II. The amount of ac phase noise generated by air-cooled or water-cooled lasers can be measured from the change in the ac phase noise in the Michelson interferometer when the cooling system of the original laser is turned on or off. In the case of air-cooled laser systems, moving the fan away from the laser head and using flexible plastic tubing to conduct the cooled air to the laser will usually reduce ac phase noise. In the case of water-cooled lasers, the simplest solution is to remove the laser from the optical table and use either fiber optic coupling or simple adaptive optics between the laser and the optical system.

(d) Choose whenever possible an optical system which is inherently less sensitive to vibrations. Such optical systems can often be built very simply using standard optical components by slightly modifying standard optical system configurations as discussed in Appendix A..$^{33-35}$ In many cases, modifying the optical system will be sufficient in itself to reduce ac phase noise to an acceptable level without carrying out the remaining steps of Sec. VI. A straightforward change in the optical system is therefore highly recommended in view of the case with which it can be carried out. As noted above, changing the type of optical system will often reduce very low-frequency phase noise (less than $1 \mathrm{~Hz}$ ).

(e) Use commercial quality vibration isolators or one of the comparably effective but much less expensive air bladder trampoline-type vibration isolators described in Appendix B to reduce the amplitude of floor vibrations transmitted to the optical table.

(f) Reduce the height of the optical components and optical mounts to a minimum. ${ }^{9,36}$ Increase the effective thickness and hence the rigidity of optical mounts (e.g., by using three rigidly interconnected rods rather than a single mounting rod) ${ }^{37,38}$ If possible try to use high-quality commercial mounts employing kinematic or gimbal-type mountings. If such commercial mounts are unavailable one should reduce the number of separate parts within the optical mount and use zero-backlash positioning screws such as those used in microwave tuners, micrometer screws, etc. ${ }^{39-44}$

Equally important is the necessity of ensuring a rigid coupling between the optical table and optical mounts as well as that between the optical components and the opti- cal mounts. In practice, one must securely bolt, glue, screw down, or fasten the optical mounts with tie-down clamps to the optical table and use similarly rigid mounting techniques for fastening optical components to the optical mounts. Loading springs for optical components should be as stiff as possible. Some care should, of course, be taken not to deform or damage optical components. Holographic objects should be fastened using easily removable bonding agents such as wax. A single drop of instant glue can be used to fasten more robust objects. Objects mounted in such a way can be removed by a light impact. The use of instant glue is especially recommended for magnetic mounts which frequently do not provide sufficiently rigid coupling to the optical table. This is especially serious for high, bulky magnetic mountings and/or thin (i.e., less than $5 \mathrm{~mm}$ thick) optical table top surfaces.

The importance of extremely rigid optical table/mount and optical mount/component coupling cannot be emphasized too strongly since even small vibrational motion in the mountings or in the optical components can completely dominate the mechanical response of the optical system (see Fig. 2).

(g) The transient vibrational response of the optical table should be observed by striking the optical table with a light hammer and listening to the high-frequency ringing audible to the ear as well as by directly feeling the lowfrequency vibrations with fingers lightly touching the optical table. ${ }^{9,37,45}$ If the response does not die out almost immediately (decay time on the order of $0.2-0.3 \mathrm{~s}$ ), and the interferometer is strongly affected by weak acoustic excitations, then it will be necessary to increase the effective dynamic rigidity of the optical table. The quickest way of increasing the effective dynamic rigidity will be to mount the optical system on a breadboard or massive metallic plate which is placed directly on the optical table or, alternatively, to place a large wooden plate between thinner optical tables (i.e., less than $15 \mathrm{~cm}$ ) and the vibration isolator. ${ }^{46-49}$ In both cases, there should be no intermediate vibration isolator layer between the additional plate and the optical table since this would greatly reduce the damping due to the additional plate. One should also make sure that the additional plate is as flat as possible in order to maximize the direct contact area between the additional plate and the optical table. This increase in damping can readily be observed as a marked decrease in the ringing of the optical table after a light impact.

\section{SUPPLEMENTARY PROCEDURE}

If the Standard Procedure is not sufficient, then it will be necessary to locate the dominant remaining source of ac phase noise. This must be carried out in the original interferometric system suitably modified, if necessary, to give an output simply related to the ac phase noise. A quick identification of the dominant noise sources can often be obtained by observing the frequency response of the phase noise in the original interferometric system by using a spectrum analyzer or a (preferably digital) oscilloscope. Any signal at the power line frequency or its multiples, which 


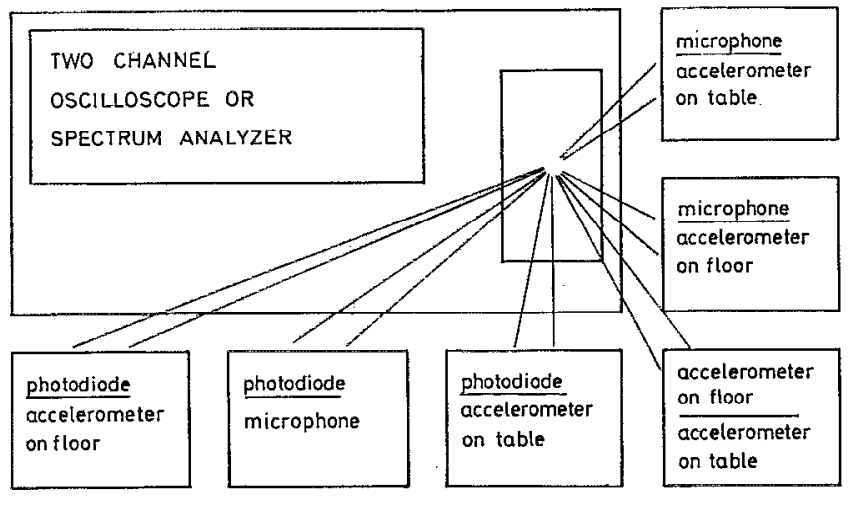

FIG. 3. Schematic illustration of the various types of simple correlation measurements which can be carried out to determine the source(s) of ac phase noise. The photodiode output gives the amplitude of the ac phase noise while the microphone output gives the amplitude of the ambient acoustic flux. Both temporal and frequency correlations between different possible noise sources and the measured ac phase noise can be found by using the oscilloscope or the spectrum analyzer, respectively. One or more of the correlation combination pairs shown in the figure can be used under existing experimental conditions or, more effectively, with an on/off technique in which the correlation measurements are compared both before and after changing some parameter in a suspected noise source, transmission path, or the optical system.

remains when one arm of the interferometer is blocked, is due to amplitude modulation of the laser and does not represent genuine phase noise. If, however, the dominant ac phase noise is monotonically increasing below $\sim 30 \mathrm{~Hz}$ and contains no periodic components, then it is very likely that residual index of refraction fluctuations are responsible. On the other hand, if the dominant ac phase noise components are quasiperiodic and lie between $\sim 10$ and $100 \mathrm{~Hz}$, or above $100 \mathrm{~Hz}$, then floor vibrations or acoustic coupling, respectively, should be suspected. If the amplitude of the noise increases while talking or singing with the character of the frequency spectrum unchanged, the dominant noise source is probably acoustic. If the noise increases with a relatively unchanged frequency spectrum while stepping and hopping on the floor, the dominant noise source will probably originate from floor vibrations. A more quantitative verification can be obtained by carrying out a simple correlation check illustrated in Figs. 3-5 where the amplitude of floor vibrations and the amplitude of the acoustic flux, measured by the accelerometer and the microphone, respectively, are compared with the photodiode output of the interferometer. It is worth emphasizing that the measurements shown in Figs. 4 and 5 are similar to measurements carried out by the authors using commercial optical tables and vibration isolators and are representative of well-built interferometric systems (table, mounts, vibration isolator) in general, whether commercial or homemade, under normal experimental conditions.

If index of refraction fluctuations are the dominant source of ac phase noise, one should use ellipsometric or other techniques insensitive to index of refraction fluctuations (see Appendix A). If this is not possible, then one can immerse the interferometer in helium gas (e.g., by placing the interferometer inside a plastic glove bag) or

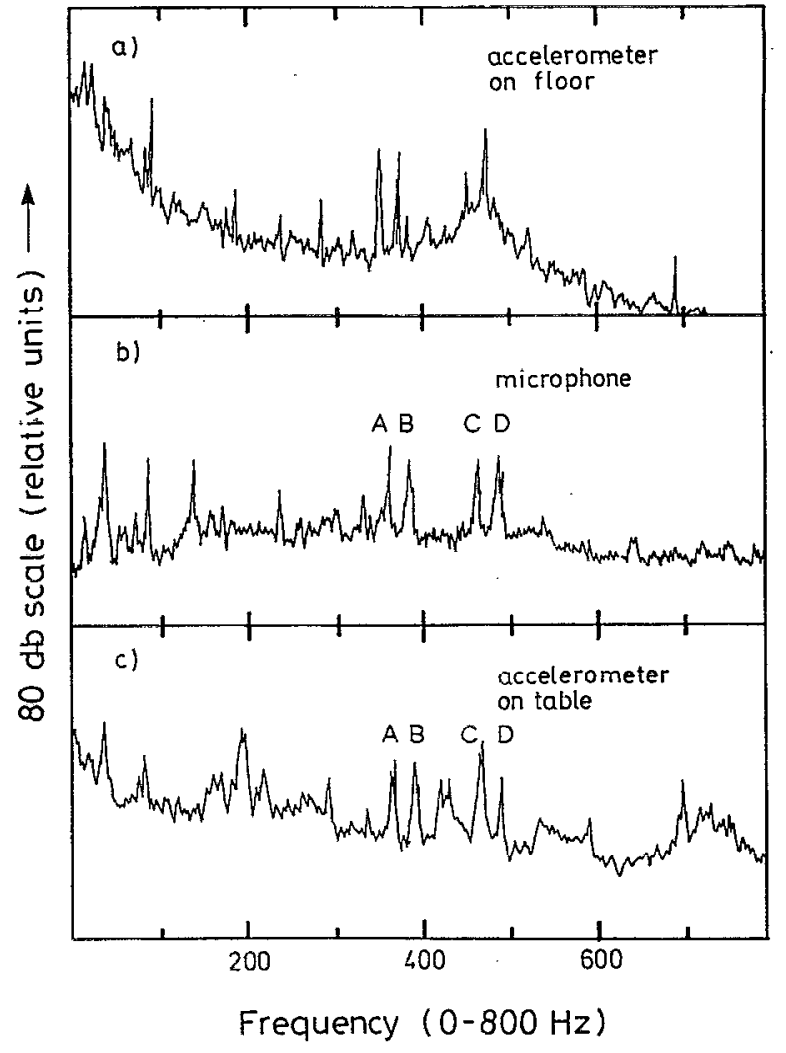

FIG. 4. (a) Measurements of the amplitude of floor vibrations, (b) the amplitude of the ambient acoustic flux, and (c) the amplitude of optical table vibrations carried out under the same experimental conditions. The optical table consisted of a Newport XS-24 breadhoard strongly damped by an auxiliary wooden plate and mounted on an air bladder vibration isolator as described in Sec. VI and Appendix B. The large reduction in the amplitude of floor vibrations transmitted to the optical table due to the presence of the vibration isolator can be immediately seen from the almost complete removal of the low-frequency seismic and broad resonance $(\sim 480 \mathrm{~Hz})$ maxima in the floor vibrations [cf. (a) and (c)]. The close general resemblance between (b) and (c), as well as the exact overlap of the sharp resonances (denoted by A, B, C, D) shows the typical strong correlation between the ambient acoustic background and optical table vibrations for vibration-isolator mounted optical systems.

place the interferometer inside a moderately evacuated vacuum container. ${ }^{50}$ If acoustic coupling is the dominant residual source of phase noise, it will be advisable to cover all doors, windows, and other opening with rock-wool type sound isolation whenever the source is suspected to lie outside the laboratory. Special care should be taken to cover the edges and corners of such openings which are usually the most serious sources of sound leaks. ${ }^{51}$ Other useful laboratory furnishings, when available without too much expense, will include standard equipment used in TV or recording studios such as acoustically isolated double doors, double-layer windows, floor carpeting, damped water and ventilation pipes, damped air conditioners, typical TV or recording studio acoustic damping, different size sound diffusers, etc.

If the noise source is suspected to lie inside the laboratory, one should turn off air conditioning, ventilation duct blowers, etc. If this is not sufficient, the most practical solution will be to damp or isolate the source of acoustic 


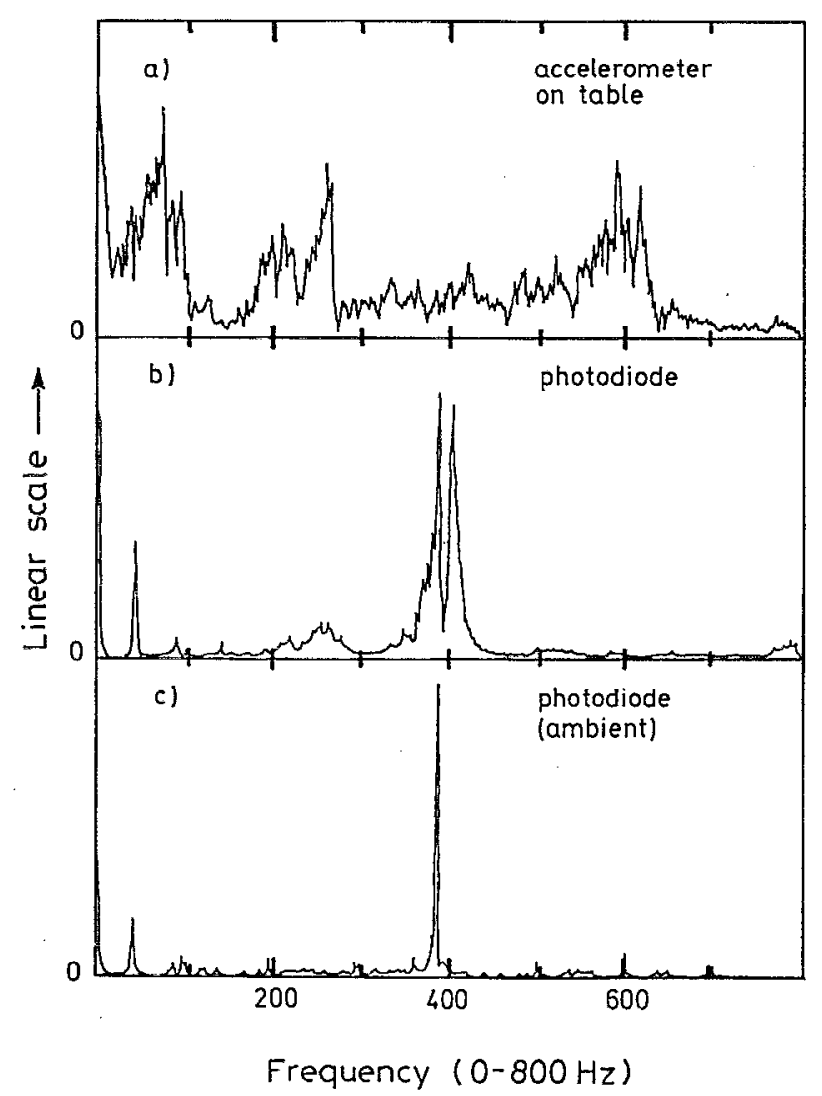

FIG. 5. (a) The amplitude of optical table vibrations and (b) the amplitude of ac phase noise given by the photodiode output under the same experimental conditions with a loudspeaker mounted close to the optical table and driven by a white noise generator as discussed in Sec. V. (c) shows the photodiode output under normal ambient experimental conditions with the loudspeaker turned off. The large peak observed at $391 \mathrm{~Hz}$ in (b) and (c) is acoustically excited by the ventilation blower of the spectrum analyzer used to record the photodiode output. The vibration isolator optical table system is the same as that used to record the measurements in Fig. 4. A comparison of (a) and (b) clearly demonstrates the relatively small effect of most optical table vibrations on ac phase noise for typical vibration isolator and optical table systems. The ac noise observed in (b) and (c) is almost exclusively due to the acoustic excitation of resonant vibrations in the optical mounts and components (391 and $407 \mathrm{~Hz}$ ), laser noise (at multiples of $50 \mathrm{~Hz}$ ), and residual index of refraction fluctuations (near $0 \mathrm{~Hz}$ ).

flux since it is extremely difficult to shield the entire optical system directly. ${ }^{52}$

If floor vibrations are the dominant source of ac phase noise, the only viable approach will be to use a more rigid optical table, active vibration isolation, or more sophisticated suspension systems such as those employed in gravitational wave detection. ${ }^{53-55}$ Further decreases in the ac phase noise will require an additional increase in the dynamic rigidity of the optical table/mount system. This is easiest to do using a frequency-swept generator connected to a loudspeaker to acoustically excite resonance vibrations of the table/mount system over the frequency range $\sim 20$ $800 \mathrm{~Hz}$. Such a procedure should be carried out first for optical mounts since the acoustic excitation of eigenmode vibrations in the optical mounts will normally lead to larger phase noise than that due to vibrations of most optical tables even when mounted on simple homemade vi-
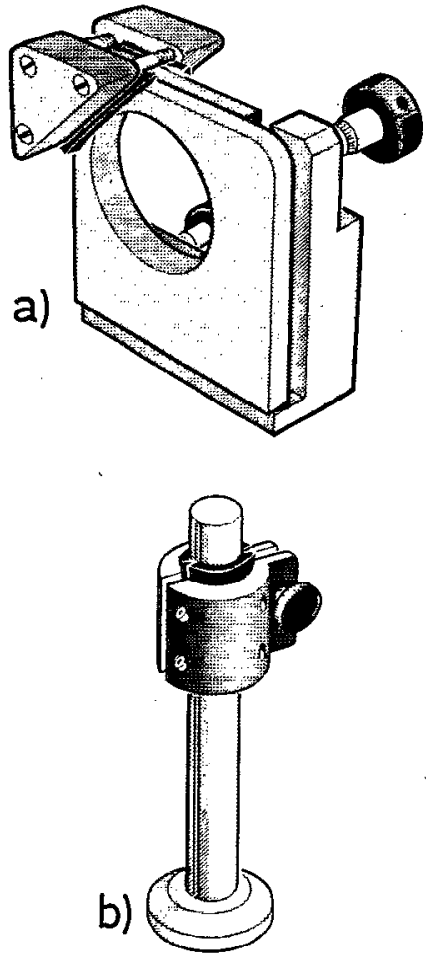

FIG. 6. (a) Simple methods of damping standard Kinematic-type mounts and (b) vertical mounting rods using an additional mass and an intermediate rubber damping layer. The additional mass should be $\sim 5 \%-40 \%$ of the mass of the mount itself. The thickness (typically a few millimeters) and type of the damping layer can be varied on a trial-anderror basis with the mechanical tension applied by the additional mass mounting screws adjusted in order to obtain the optimal damping.

bration isolators. ${ }^{56}$ This can be done relatively simply using a quick trial-and-error approach by fastening a mass which is an appreciable fraction (5\%-40\%) of the mass of the mounting to the mounting through an intermediate rubber damping layer (see Fig. 6), and adjusting the mechanical tension of the fastening screws to obtain maximum reduction in the phase noise. ${ }^{46-49,57}$

Phase noise due to optical table eigenmode resonance vibrations at frequencies above $100 \mathrm{~Hz}$ can be effectively reduced by placing relatively small masses $(<10 \mathrm{~kg})$ directly on the top surface of moderately damped optical tables $\left(Q_{\text {table }}>20\right) .{ }^{51}$ For lower-frequency resonance vibrations, practical considerations will usually require that larger masses be placed between the optical table and the vibration isolators with a separate mass for each vibration isolator

For workers who must build a homemade optical table or who need a highly damped optical table $\left(Q_{\text {table }}<10\right)$, we recommend using the same triple layer, sandwich, construction used in commercial optical tables with the middle honeycomb layer replaced by specially treated construction wood with 5-mm-thick metal plates used for the top and bottom surfaces. ${ }^{57,58}$ Such homemade optical tables have approximately the same density as common honeycomb optical tables but are much easier to construct, demount, and design to meet special requirements. The metal-woodmetal structure ensures good dynamic rigidity with good 
mounting facilities. If it is too expensive, or heavy to use a 5-mm-thick metal tabfe top, then somewhat thinner top and bottom plates can be used or even omitted with mounts strongly screwed into the wood with only a slight degradation in performance for thicker tables. ${ }^{59}$

Lastly, we note that the dimensions of optical tables and interferometric systems should be kept as small as convenient, viz. Fig. 1. The use of optical tables with smaller transverse dimensions will often be helpful by re ducing the amplitude of optical table resonances while the use of smaller interferometric systems will be helpful in reducing the relative rotations of optical components. ${ }^{36,38}$

\section{ACKNOWLEDGMENTS}

The authors wou数 like to thank Kurt Omø, Ib Rasmussen, Torben Riisbo, Rurt Andersen, and Torben Nielsen for the loan of instruments used to carry out measurements in this work. The authors would also like to acknowledge Mogens Ohlrich and Tomasz Lekszycki for helpful discussions, and Ove Broo Sørensen for the threedimensional figures.

\section{APPENDIX A: MODIFYING THE OPTICAL RESPONSE}

We restrict our attention here to double-beam ctrcular interferometer (DBCI) optical systems which will be treated in some detail since they can be implemented very easily with standard optical components by slight changes in common interferometric systems but have been treated only very briefly in the literature. ${ }^{31-33}$ Such D'BCI systems can give a reduction of ac phase noise by a factor of up to $10-10^{2}$ in essentially all interferometric systems having a small optical path difference, and hence con be used to greatly improve most two-beam interferometers, holographic setups, four-wave mixing systems, etc.

The DBCI optical system can be regarded as a slight modification af the ordinary two- and three-mirror common path circular interferometer (e.g., Sagnac interferometer) where the two beams are spatially separated in contrast to the common path circular interferometers where both beams overlap. Thus, it is possible to place an object under study in one beam and to introduce a variable independent phase delay in the other beam, e.g., by using a simple refractive element. Since the two beams are spatially separated in the DBCI, ac phase noise due to index of refraction fluctuations will still be present. However, by the proper choice of optical components and DBCI geometries, the mechanical response of DBCI systems to vibrational excitations [viz. the factor $R_{\text {opt }}$ Eq. (3)] will be much smaller than for other more conventional two-beam interferometers (e.g., standard Michelson and MachZelonder interferometers). The greatly increased stability of DBCI optical systems compared to conventional twobeam interferometers results from the fact that there will be no change in the ac phase due to translational motion of optical components in DBCI systems and (usually) only second-order changes in the ac phase due to rotational notion of the optical components. ${ }^{33-35}$ In practice, this means that both the DBCI and common path circular in-
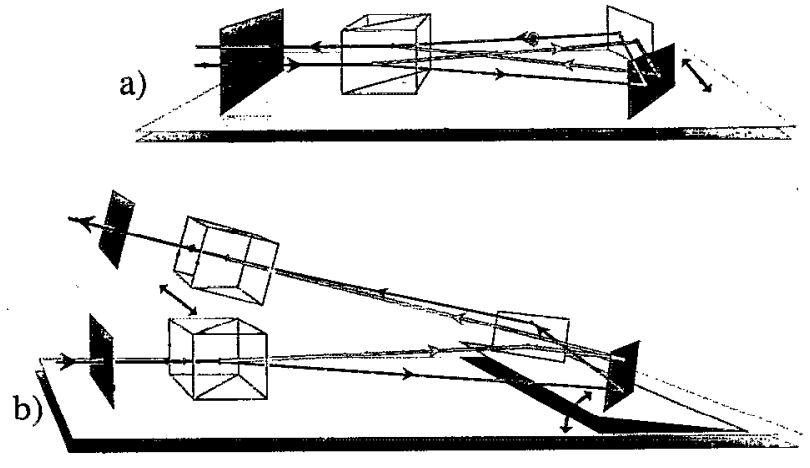

FIG. 7. Extremely stable DBCI configurations employing linearly polarized incident light and a Wollaston prism as a polarizing beam splitter. (It should be roted that the beam splitters and beam combiners for all the DBCI configurations shown here and in Figs. 8-10 can be replaced by reflection diffraction gratings for applications using monochromatic light as discussed is the text). A $45^{\circ}$ Wollaston prism should be used such that the two beams leaving the prism (single- and double-line beams) are polarized at $\pm 45^{\circ}$ relative to the plane containing the two beams. The mirrors should be rigidly mounted together with the normal to the mirrors parallel (or nearly parallel) to the common plane of the light beams. Reflection at the two mirrors will then introduce an additional $90^{\circ}$ relative change in the polarization directions of the two beams making it unnecessary to use a wavelength-dependent half-wave plate to obtain interference. The extreme stability of such DBCI configurations results from the fact that rotations about the common optical axis of such interferometers are the only motions which can leas to a shift in the interference fringe pattern. The input and output beams can be separated horizontally in (a) and vertically in (b) by a parallel displacement of the two mirrors shown in (a) (straight double arrow), or by rotating the two mirrors shown in (b) (curved double arrow). The double-Wollaston DBCI configuration shown in (b) makes it possible to change the interference amplitude ratio by more than two orders of magnitude by rotating the polarizer or the analyzer. The phase difference between the two beams in (b) can be independently varied by a relative parallel displacement of the prisms indicated by the straight double arrow. Such a parallel displacement can be carried out using a high-quality translation stage and micrometer screw.

terferometers will function as inherently zero-order interferometers readily forming white light fringes and possessing excellent fringe stability.

We present here only a brief outline of DBCI optical systems in order to illustrate the very large number of DBCI which are possible. The most stable DBCI system considered here will contain one or two adjustable rigidly mounted Wollaston prisms as shown in Fig. $7{ }^{60}$ The extremely large stability can be dramatically demonstrated by observing white light fringes which are easily seen even when the Wollaston prisms are hand held. A very useful property of such a DBCI interferometer is the fact that one can easily change the relative intensities of the two beams by at least four orders of magnitude. The relative displacement of the two prisms can be used to measure the phase difference between the two beams. The spatial separation of the beams can be increased if desired by using two folding mirrors. The angular separation of the beams for a quartz Wollaston prism is of the order of $1^{\circ}-2^{\circ}$.

Figure 8 shows several DBCI optical systems employing ordinary and polarizing beam splitters which can replace Mach-Zehnder interferometers in almost all applications with the factor limiting the maximum beam. 


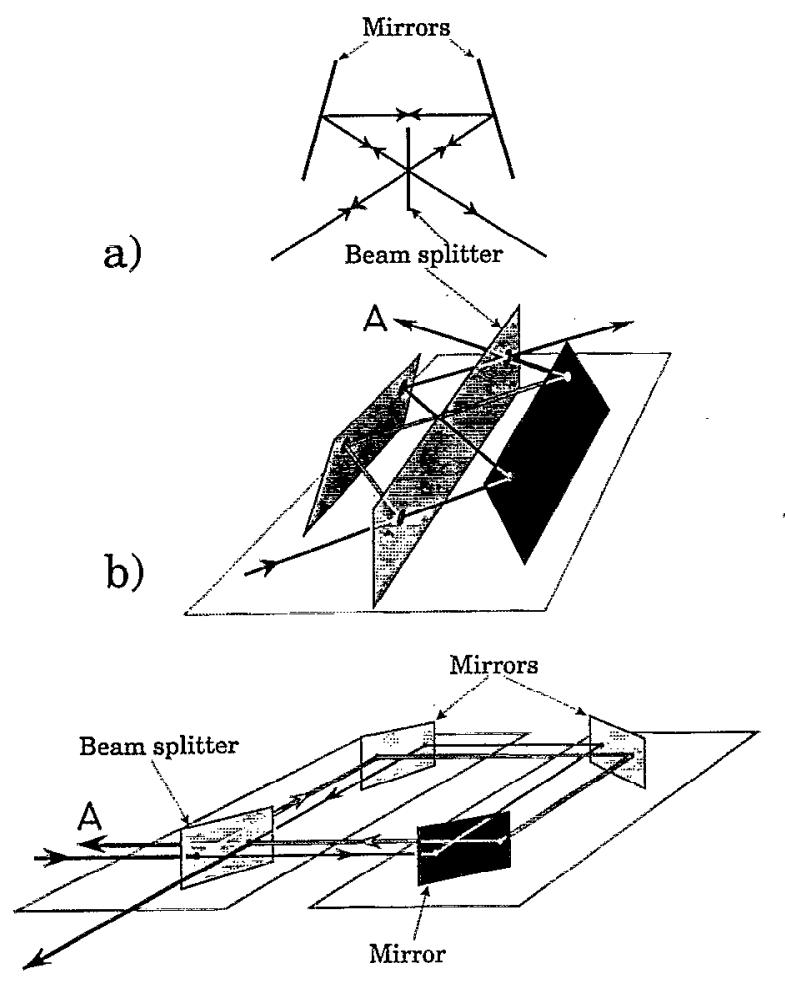

c)
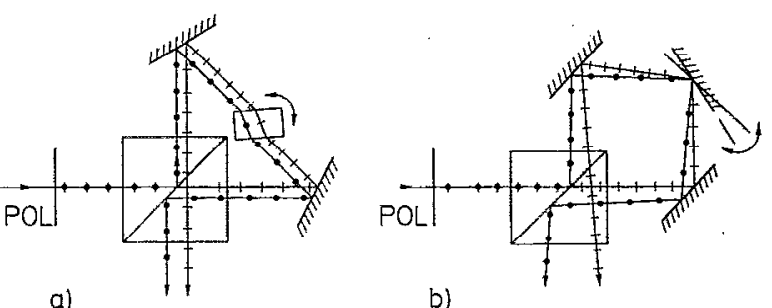

b)

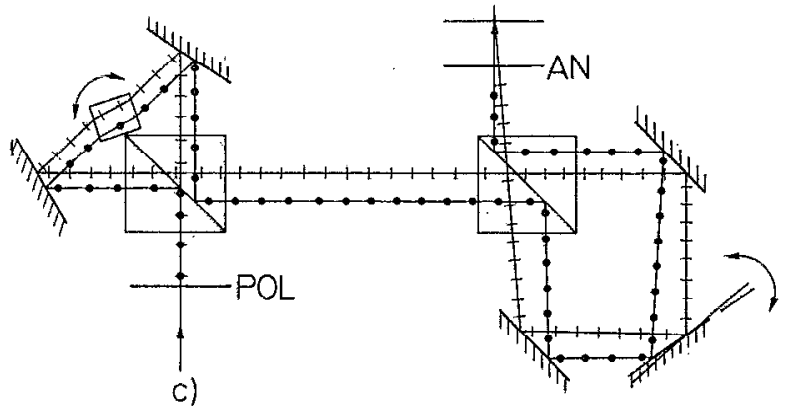

FIG. 9. The rigid configurations employing polarizing beam splitters in (a) and (b) function in the same way as a calcite plate and a Wollaston prism permitting an independent variation of the spatial separation between the beams [by rotating the plane-parallel glass plate in (a)] as well as the angle between the beams [by rotating the center mirror in (b)]. The two functions are combined in the DBCl configuration shown in (c).

ized directly on detectors, e.g., a multichannel analyzer, a linear array, etc. Such DBCI systems will be well suited to low-resolution FFT spectroscopy. It is worth noting that the configuration shown in Fig. 9(a) can be used to replace the two-slit source in modified Young experiments resulting in considerable increase in intensity even with extended light sources.

The DBCI geometry can also be used to improve holographic systems which are in fact equivalent to generalized interferometers with one mirror replaced by the holographic object and the beam combiner replaced by the photographic plate. One such holographic DBCI configuration is shown in Fig. 10. The holographic object must, of course, be rigidly connected to the reference beam mirror.

It is worth pointing out that reflection type diffraction gratings (both ruled and holographic) can be used to replace the beam splitters and beam combiners in each of the DBCI systems above for applications using monochromatic light. Such grating DBCI constructions avoid problems associated with nonparallelism, optical inhomogeneities and limited substrate transparencies (especially for UV or IR radiation) of plate-type, cube-type, and Wollaston-type beam splitters, leading to extra stable interferometers with very high fringe contrast ratios $\left(C>10^{3}\right){ }^{61}$ The input and output beams of such grating DBCI should be normal or nearly normal to the grating with the two first-order diffracted beams directed along the two arms of the interferometer. The blaze direction of the grating should be oriented to give the same intensity of the two interfering beams. Such grating DBCI systems can also be used as extremely stable phase grating moiré pattern interferometers with intensity changes at the output introduced by a parallel relative displacement of the grat- 

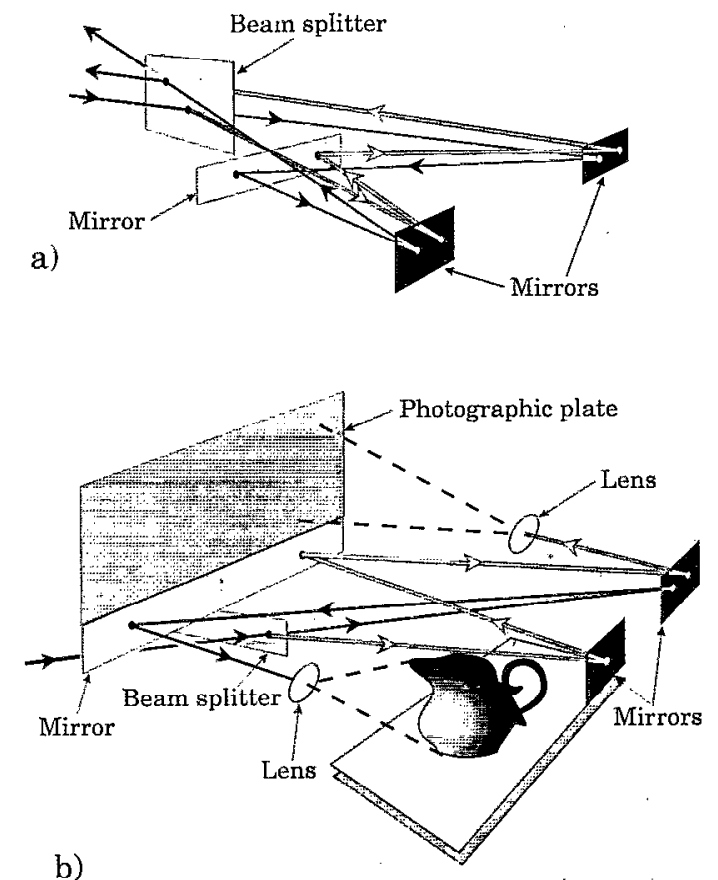

FIG. 10. (a) and (b) show how a simple three-mirror DBCI configuration can be modified to obtain a stable holographic setup. Part of one of the mirrors in (a) is replaced by the holographic object while part of the beam splitter is replaced by the recording medium, e.g., photographic plate, photorefractive crystal, etc. The holographic object must be rigidly fixed to the mirror while the photographic plate must be rigidly fixed to the beam splitter. Such a double-beam holographic configuration provides a stable control of beam intensities.

ings perpendicular to the groove direction..$^{62} \mathrm{~A}$ displacement of the grating by one grating period $(0,83 \mu \mathrm{m}$ for a 1200 lines/mm grating) will introduce a $4 \pi$ phase shift. Fringe counting and fringe interpolation techniques make it possible to carry out distance and displacement measurements with subnanometer resolution. Since the measured displacement is determined only by the grating periodicity and is not directly related to the wavelength, expensive stabilized single-frequency sources are not needed. Low thermal expansion Zerodur substrate gratings can be used for higher accuracy.

\section{APPENDIX B: VIBRATION ISOLATOR}

In our experience, home-made air bladder systems are invariably used incorrectly with a greatly degraded performance as compared with commercial systems. It is absolutely necessary that the resonance frequency $\Omega_{0}$ of the table mounted on the air bladder be much less than the characteristic frequencies of floor vibrations as can be seen from the approximate equation for the amplitude of the floor acceleration, $a_{\text {floor }}$, transmitted to the optical table

$$
a_{\text {table }}=\frac{a_{\text {floor }}}{1-\left(\omega / \Omega_{0}\right)^{2}} \rightarrow\left(\frac{\Omega_{0}}{\omega}\right)^{2} a_{\text {floor }} \text { for } \omega>\Omega_{0} .
$$

At or close to the resonance frequency $\left(\omega \approx \Omega_{0}\right)$ the vibration isolator will amplify the amplitude of floor vibrations transmitted to the optical table by the $Q$ factor of the vi- bration isolator itself. ${ }^{63}$ Below the resonance frequency $\left(\omega<\Omega_{0}\right)$ the vibration isolator gives no reduction in the acceleration transmitted to the optical table. In practice, this means that the resonance frequency $\Omega_{0}$ should be below $5 \mathrm{~Hz}$.

The air bladder vibration isolator can be modeled by a parallel combination of two unidirectional springs where one spring represents the elasticity $K_{\text {air }}$ of the enclosed air, and the other spring represents the elasticity $K_{\text {bladder }}$ of the flexible membrane surface. The resonant frequency $\Omega_{0}$ of such an air bladder can therefore be written as ${ }^{64}$

$$
\Omega_{0}=\sqrt{K_{\text {air }} / M+K_{\text {bladder }} / M},
$$

where $K_{\text {air }}$ is the effective spring constant of the enclosed air given by

$$
K_{\text {air }}=\frac{\sigma A^{2}}{V_{0}}\left(P_{0}+\frac{M g}{A}+P_{K}\right) .
$$

Here $\sigma$ is the specific heat ratio for air, $A$ is the effective cross-sectional area of the bladder surface in contact with the optical table supports, $V_{0}$ is the volume of air inside the air bladder, $M$ is the mass of the optical table plus optical mounts and components while the parenthesis in Eq. (B3) gives the total air pressure in the air bladder where $P_{0}$ is the unloaded air pressure inside the air bladder, $(M g / A)$ is the weight-induced excess pressure, and $P_{K}$ is the surfacetension-induced internal pressure.

The bladder membrane surface is responsible for most of the damping of the air bladder system and provides vibration isolation in three dimensions for the toroidal inner tube geometry most commonly employed in homemade air bladder systems. ${ }^{65}$ On the other hand, the elasticity of the bladder surface has the disadvantage of increasing the resonance frequency through the factors $K_{\text {bladder }}$ and $P_{K}$ in Eqs. (B2) and (B3), both of which are functions of the effective contact area. Thus the most common mistakes made by workers employing homemade air bladder systems are the use of an effective contact area $A$ which is excessively large, and the use of relatively small or thick inner tubes such that the bladder elasticity is significantly greater than that of the enclosed air. As a result, the resonance frequency of most homemade air bladder systems is so high ( $10-15 \mathrm{~Hz}$ or higher) that vibration isolation against floor vibrations is severely compromised with a greatly increased risk of transmitting and amplifying subsonic floor vibration resonances. It has been the authors' experience that it is extremely difficult to reduce the loaded resonance frequency of a vibration isolator system employing separated inner tubes to less than about $5 \mathrm{~Hz}$ even when the opposing requirements of minimum contact area and large soft air bladders are fully optimized for available simple air bladder types (e.g., car, truck, bicycle, basketball, football, bladders, etc.). Since a resonance frequency of $5 \mathrm{~Hz}$ may not always be sufficiently low (especially for optical systems which are not very rigid) we recommend one or more of the trampoline-type suspended inner tube constructions shown in Fig. 11 which are both easy to construct, move, demount, etc. and very inexpensive. Such trampoline-type inner tube constructions greatly reduce 
a)

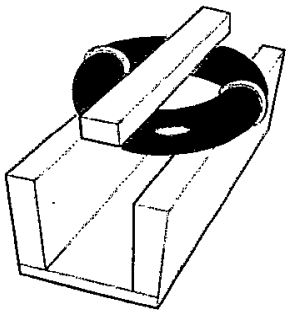

b)

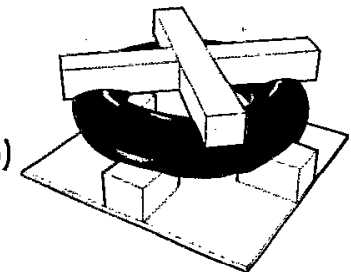

c)

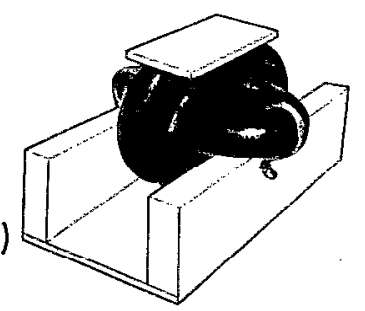

FIG. 11. Different suspended trampoline-type vibration isolator configurations employing one or more truck, automobile, or racing bicycle inner tube/tire combinations. The horizontal inner tube/tire combinations shown in (a) and (c) must be bound by restraining bands to maintain proper inner tube suspension. (a) and (b) show low-height constructions which can be built very simply (a) or are especially well suited to lowvolume high-pressure air bladders (b) such as those employed on racing bicycles where the inner tube and tire are permanently glued and sewn together. The mutually interacting inner tube construction shown in (c) provides the lowest vibration isolator resonance frequency $(<2 \mathrm{~Hz})$.

the effective stiffness of the rubber-like material of the inner tubes making it possible to obtain a loaded resonance frequency comparable to that of commercial pneumatic vibration isolators, $(1-2 \mathrm{~Hz}$ ), for a typical $50 \mathrm{~kg}$ mass supported by three car inner tubes. ${ }^{66-68} \mathrm{~A}$ system of three or more such inner tube constructions will support a typical optical table giving excellent vibration isolation in both the horizontal and vertical directions. Such constructions can also be made more compact than most commercial vibration isolators and, when racing bicycle combined inner tube/ tire air bladders are used, provide a very stable support without the disadvantage of automatic leveling systems since the gradual loss of air in a single inner tube will not greatly affect the stability of the entire inner tube construction. Such racing bicycle inner tube/tire combinations can be used at relatively high pressures (up to $10 \mathrm{~atm}$ ) allowing larger loads to be supported with a relatively small vibration isolator air volume. The resonance frcquency damping of such homemade trampoline-type vibration isolators is not as high as that of some commercial vibration isolators, but is not a significant factor in practice for most optical systems which are insensitive to the essentially rigid-body motion of optical tables at very low $(1-2 \mathrm{~Hz})$ vibration isolator resonance frequencies.
${ }^{1}$ Many of the most robust optical instruments (e.g., optical microscopes) are, in fact, interferometric systems as pointed out by Zernicke: Nobel Lectures Physics 1942-1962 (Elsevier, Amsterdam, 1964), p. 241.

${ }^{2}$ Annual Reference Catalog for Optics, Science and Education (Edmund Scientific, Barrington, New Jersey, 1993).

${ }^{3}$ Many of the techniques described will be especially useful for teaching laboratories, schools, or for workers on a limited budget. A number of the procedures discussed will also be helpful in improving the stability of noninterferometric systems such as scanning tunneling microscopes and other vibration sensitive instruments.

${ }^{4}$ M. M. Malley, G. W. Sutton, and N. Kincheloe, Appl. Opt. 31, 4440 (1992).

${ }^{5}$ H. Matsumoto and K. Tsukahara, Appl. Opt. 23, 3388 (1984).

${ }^{6} \mathrm{G}$. A. McBean and J. A. Elliot, Boundary-Layer Metrology 20, 101 (1981).

${ }^{7}$ Index of refraction fluctuations due to changes in the pressure or the humidity are usually much smaller than the changes induced by ambient temperature vibrations and will normally not be important at the frequencies ( $1 \mathrm{~Hz}$ or above) considered in this paper. A. Ernst, Digital Linear and Angular Metrology, 2nd ed. (Verlag Moderne Industrie AG, 1992); D. A. Wolf, Optics and Laser Technology, pp. 29-36, Feb. 1979.

${ }^{8}$ Handbook of Acoustical Measurements and Noise Control, 3rd ed., edited by C. M. Harris (McGraw-Hill, London, 1991).

${ }^{9}$ Shock and Vibration Handbook, 3rd ed., edited by C. M. Harris (McGraw-Hill, New York, 1987).

${ }^{10}$ Challenge Vibration Herz Catalog (1993) Herz Industry Co., Fujisawa City Japan, U.S.A. distributor, 22681 Via Santa Maria, Mission Viejo, CA.

11 TMC Catalog Nos. MG 37-90, MG 36-91 (1991), Technical Manufacturing Corporation, Peabody, MA.

${ }^{12}$ Y. Kuk and P. J. Silverman, Rev. Sci. Instrum. 60, 165 (1989).

${ }^{13}$ M. Okano, K. Kajimura, S. Wakiyama, F. Sakai, W. Mizutani, and M. Ono, J. Vac. Sci. Technol. A 5, 3313 (1987).

${ }^{14}$ Yu. I. Ostrovsky, M. M. Butusov, and G. V. Ostrovskaya, in Springer Series in Optical Sciences, Vol. 20, edited by D. L. MacAdam (Springer, Berlin, 1980).

${ }^{15}$ E. N. Gilbert, Acustica 66, 290 (1988).

${ }^{16}$ T. D. Rossing, Am. J. Phys. 44, 1220 (1976).

${ }^{17}$ L. Crescentini and G. Renzella, Rev. Sci. Instrum. 62, 1206 (1991).

${ }^{18}$ G. Doswell and H. Kunov, Rev. Sci. Instrum. 61 (7), 1986 (1990).

${ }^{19}$ R. G. White and D. C. Emmony, J. Phys. E 18, 658 (1985).

${ }^{20} \mathrm{P}$. Hariharan, Basics of Interferometry (Academic, London, 1992).

${ }^{21}$ The liquid and the working temperature should be chosen to minimize $d n / d T$.

${ }^{22}$ It is worth noting that the factor $R_{\text {opt }}$ will be very sensitive to the type of interferometric system employed as well as to the type of mechanical vibration excited.

${ }^{23}$ P. Hariharan, Optical Interferometry (Academic, Sydney, 1985).

${ }^{24}$ W. H. Steel, Interferometry, 2nd. ed. (CUP, Cambridge Studies in Modern Optics 1, London, 1983).

${ }^{25}$ E. Fortin and D. Singh, Am. J. Phys. 59, 891 (1981).

${ }^{26}$ Instrumentation for Sound and Vibration, etc. Catalog (Bruel and Kjaer, Naerum, Denmark, 1991).

${ }^{27}$ T. D. Rossing and D. A. Russel, Am. J. Phys. 58, 1153 (1990).

${ }^{28}$ Index of refraction fluctuations due to the pressure vibrations generated by typical HiFi loudspeakers $(60 \mathrm{~dB})$ will lead to much smaller changes in the phase $\left(\Delta n / n \sim 10^{7}\right)$ than the phase change due to resonance vibrations of the optical components excited by the loudspeaker, see Fig. 5(b).

${ }^{29}$ Temperature gradients in the laser cavity will introduce a modulation of the laser frequency leading to a decrease in the effective coherence length.

${ }^{30}$ B. Stahl, Acustica 63, 42 (1987) (in German).

${ }^{31}$ R. Latorre, Acustica 60, 77 (1986).

${ }^{32}$ Such an enclosure will also be very helpful in eliminating slowly varying $(<1 \mathrm{~Hz})$ pressure-induced variations in the refractive index leading to severe stability problems in open interferometric cavities.

${ }^{33}$ I. Filinski, Int. Sym. Appl. Opt. 1991 Aplikavana Optika 91, 136 (1991).

${ }^{34}$ I. Filinski and T. Skettrup, Prov. ICO 15 Optics in Complex Systems, SPIE 1319, 256 (1990).

${ }^{35}$ I. Filinski, Proceedings of the International Conference, "From Galileo's occhialino to optoelectronics; frontiers of optical systems and materi- 
als," University of Padova, Italy, June 9-12, 1992, edited by P. Mazzoldi (World Scientific, London, 1993), p. 506.

${ }^{36}$ The change in the geometric path difference between optical components can be written as the sum of terms which are linear and cubic in the height of the optical components above the table top due to vibrations of the optical table itself and to the vibrations of the optical components relative to the table, respectively. See also E. Skudrzyk, Simple and Complex Vibration Systems (Penn State University, London, 1968).

${ }^{37}$ L. Cremer, M. Heckl, and E. E. Ungar, Structure-Borne Sound (Springer, Berlin, 1988).

${ }^{38}$ The exact dimensional dependence of the vibration amplitude is very complicated for most real optical systems being a sensitive function of boundary conditions, material asymmetries, damping, configuration (e.g., honeycomb, multilayer plate), etc. However, it will almost always be advantageous to choose optical tables and mountings (mounts and components) which are as small and thick as possible whenever practical. A rough indication of the type of dimensional dependences which may be found is provided by the idealized analytically solvable case of flexural vibrations in a thin homogeneous simply supported plate where the amplitude of mechanical vibrations is inversely proportional to the thickness and directly proportional to the fourth power of the transverse dimensions of the plate.

${ }^{39}$ New Focus, Inc. Catalog (1993), Mountain View, CA.

${ }^{40}$ Thorlabs Inc. Catalog (1993), Newton, NJ.

${ }^{41}$ Newport Catalog (1993), Irvine, CA.

${ }^{42}$ K. C. Hawkins and H. N. Rutt, Opt. Laser Technol. 21, 125 (1989).

${ }^{43}$ A. W. Hartman, Rev. Sci. Instrum. 59, 2617 (1988).

${ }^{44}$ H. Damany and P. Laporte, Rev. Sci. Instrum. 59, 990 (1988)

${ }^{45}$ Y. Kubota and E. H. Dowell, J. Sound Vibration 106, 203 (1986).

${ }^{46}$ U. V. Tambe, J. Sound Vibration 124, 391 (1988).

${ }^{47}$ T. L. Smith, K. Rao, and I. Dyer, Noise Control Engineering Journal, March-April 1986, p. 56

${ }^{48}$ A. Yildiz and K. Stevens, J. Sound Vibration 103, 193 (1985).

${ }^{49}$ A. Trochidis, Acustica 51, 201 (1982) (in German).

${ }^{50}$ Both procedures will reduce the factor $(n-1)$ appearing in Eq. (1), while the substitution of helium gas for air at the same pressure will also reduce the magnitude of thermal gradients responsible for index of refraction fluctuations due to the much larger thermal conductivity of helium.

${ }^{51}$ It should be noted that optical tables mounted on vibration isolators will be very sensitive to acoustic coupling since the vibrational energy of such freely floating tables can no longer be effectively transferred to the floor and must be dissipated almost entirely by internal friction; $G$. Rosenhouse, Acustica 58, 189 (1985).

${ }^{52}$ D. Hils, J. E. Faller, and J. L. Hall, Rev. Sci. Instrum. 57, 2532 (1986).

${ }^{53}$ A. Giazotto, D. Passuello, and A. Stefanini, Rev. Sci. Instrum. 57, 1145 (1986).

${ }^{54}$ S. A. Paipetis and A. F. Vakakis, J. Sound Vibration 98, 13 (1985).

${ }^{55}$ P. Lorrain, Rev. Sci. Instrum. 50, 664 (1979).

${ }^{56}$ This will always be true with the exception of unusually large amplitudes of floor vibration or a large overlap in the resonance response of the optical table/mount system and that characteristic of floor vibrations. It should be noted that the damping of optical mounts will greatly facilitate the tuning of interferometers by rapidly reducing the large transient vibrations which are always introduced when adjusting optical components.
${ }^{57}$ Damping can be due to a combination of different mechanisms including a simple mass loading of vibrational eigenmodes, an auxiliary mass damping due to a phase-reversed opposing motion of the additional mass, and a mechanism by which vibrational energy is resonantly coupled out of the optical table or mountings into the additional massintermediate layer vibrational system.

${ }^{58}$ The use of wooden veneer blocks in which the individual layers are sorted to remove knots and carefully dried and bonded together under pressure at high temperatures using a waterproof formaldehyde-base glue can be highly recommended. Such woods possess a dynamic rigidity comparable to that of steel permitting the construction of rigid readily demountable highly damped optical tables $(Q<10)$ when used in cross-blocked configurations. Woods of this type also exhibit very small moisture dependent deformations. One such wood is marketed under the name "Kerto" by Metsäliiton Tedlisuus DY, Finland; Danish distributor: Kristian Staerk A/S, Søborg, Denmark.

${ }^{59}$ The optical mounts can also be rigidly fastened to small 5-mm-thick metal plates which are themselves securely screwed to the wood table surface.

${ }^{60}$ M. Francon and S. Mallick, Polarization Interferometers: Applications in Microscopy and Macroscopy (Wiley, London, 1971).

${ }^{61}$ It is worth noting that a reflection grating can be used with a single mirror as a one-arm degenerate Michelson interferometer for distance and coherence length measurements. The incident beam and mirror of such an interferometer should be oriented such that the diffracted output beams are normal to the grating.

${ }^{62}$ A. J. Durelli and V. J. Parks, Moire Analysis of Strain (Prentice-Hall, New Jersey, 1970).

${ }^{63}$ This is in fact the case for all homemade vibration isolators which the authors have encountered including that originally built by the authors. The reduction in the amplitude of floor vibrations transmitted to the optical table will be much less than that suggested by Eq. (1) at frequencies above $30-100 \mathrm{~Hz}$ where the rigid body motion assumed in the derivation of Eq. (1) no longer obtains. See also Newport Vibration Control (1993) Catalog, Irvine, CA.

${ }^{64}$ B. I. Bachrach and E. Rivin, J. Sound Vibration 86, 191 (1983).

${ }^{65}$ Common air bubble packing materials can be used as a cheap ad hoc alternative to inner tube air bladders but are significantly stiffer and hence much less suitable as vibration isolators; J. K. Severn and D. N. Batchelder, J. Phys. E 17, 113 (1984).

${ }^{66}$ No claim to originality in the use of trampoline type constructions is claimed. The effect of trampoline-like inner tube configuration is similar to that of rolling diaphragms used in commercial vibration isolators in that they allow very large changes in air volume to be obtained with only a small amount of work performed against the air bladder surface tension. Such trampoline-type constructions are discussed in some detail here since, to the author's knowledge, they are not adequately described anywhere in the literature, but can provide an effective inexpensive alternative to commercial air-spring vibration isolators if they are correctly built.

${ }^{67}$ D. L. Platus, Machine Design, February 26, 1993, pp. 123-130.

${ }^{68}$ It is important that the load not lead to a collapse of any of the inner tubes or to contact with the floor or the optical table supports which will greatly increase the resonance frequency of the vibration isolator. A belt or cord (preferably rubber) can be used to hold the suspended inner tubes under static tension in order to prevent both slow collapse and floor contact. 